\title{
Dependence of short and intermediate-range order on preparation in experimental and modeled pure a-Si
}

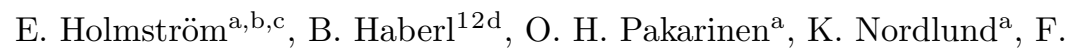

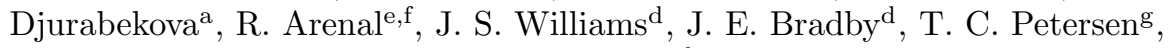 \\ A. C. Y. Liu ${ }^{\mathrm{g}, \mathrm{h}}$ \\ ${ }^{a}$ Department of Physics and Helsinki Institute of Physics, University of Helsinki, P.O. Box \\ 64, Helsinki FIN 00014, Finland \\ ${ }^{b}$ Department of Earth Sciences, University College London, Gower Street, London WC1E \\ $6 B T, U K$ \\ ${ }^{c}$ Department of Applied Physics, COMP Centre of Excellence, Aalto University, P.O. Box \\ 11100, 00076 Aalto, Espoo, Finland \\ ${ }^{d}$ Department of Electronic Materials Engineering, Research School of Physics and \\ Engineering, Australian National University, Canberra, ACT 0200, Australia \\ ${ }^{e}$ Laboratorio de Microscopias Avanzadas (LMA), Instituto de Nanociencia de Aragon \\ (INA), University of Zaragoza, E-50018 Zaragoza, Spain \\ ${ }^{f}$ Fundacion ARAID, E-50004 Zaragoza, Spain \\ ${ }^{g}$ School of Physics and Astronomy, Monash University, Clayton, VIC 3800, Australia \\ ${ }^{h}$ Monash Centre for Electron Microscopy, Monash University, Clayton, VIC 3800, Australia
}

\begin{abstract}
Variability in the short-intermediate range order of pure amorphous Si synthesized by different experimental and computational techniques is probed by measuring mass density, atomic coordination, bond-angle deviation, and dihedral angle deviation. It is found that there is significant variability in order parameters at these length scales in this archetypal covalently bonded, monoatomic system. This diversity strongly reflects preparation method and thermal history in both experimental and simulated systems. Where experiment and simulation do not quantitatively agree, this is partly due to inherent differences in analysis and time scales. Relaxed forms of amorphous Si quantitatively match continuous random networks generated by a hybrid method of bond-switching Monte Carlo and molecular dynamics simulation. Qualitative trends were identified in other experimental and computed forms of a-Si. Ion-implanted a-Si's are less
\end{abstract}

\footnotetext{
${ }^{1}$ Electronic mail: bianca.haberl@gmail.com

${ }^{2}$ Current address: Chemical and Engineering Materials Division, Oak Ridge National Laboratory, Oak Ridge, TN 37831, USA
}

Preprint submitted to Journal of Non-Crystalline Solids

January 26, 2016

(C) 2016. This manuscript version is made available under the Elsevier user license http://www.elsevier.com/open-access/userlicense/1.0/ 
ordered than the relaxed forms. Preparation methods which narrowly avoid crystallization such as experimental pressure-induced amorphization or simulated melt-quenching result in the most disordered structures. As no unique form of amorphous Si exists, there can be no single model for the material.

\section{Introduction}

Amorphous solids encompass a rich field of physics and a wealth of existing and prospective technological applications [1. Amorphous silicon (a-Si) is of fundamental interest as an archetypal tetrahedral covalent amorphous network and a major technological material for thin-film electronics [2, 3]. Other tetrahedral amorphous materials such as diamond-like carbons and amorphous chalcogenides have many uses as superhard wear-resistant coatings [4, 5, 6] and optical components [7]. Disordered materials with a tetrahedral network are also of central importance to geophysics and planetary science (e.g. liquid silicates in the interior of planets [8, 9] and low-density amorphous ice [10]). Understanding structural diversity in tetrahedral networks with no long-range ordering is thus a topic of strong scientific and technological significance. However, a robust microscopic understanding has proven elusive, and further work addressing this problem is clearly required.

Pure a-Si is an excellent model amorphous material that can be prepared reproducibly by multiple methods [11]. Despite long-standing experimental and theoretical interest in pure a-Si, an accepted model for its structure has not been established [12, 13. This is largely due to the fact that amorphous structures are characterized by correlation functions that encompass the statistical variation in atomic order in the material. To date, these correlation functions have not been measured to sufficiently high order and with sufficient precision to unambiguously identify a preferred structural model for a-Si [14]. In general it is thought that the short-range order (SRO) up to length scales of a few $\AA$, or nearest-neighbor bonding of these materials, is dominated by $\mathrm{sp}^{3}$ hybridization. Ordering on approximately the length scale of 4 to $6 \AA$ is often 
termed intermediate-range order (IRO) [15]. Beyond IRO, the extent and nature of any medium-range order (MRO) is contested and ranges from very little in the case of the continuous random network (CRN) model [16] to 1-2 nm grains with topologically crystalline order in the case of the "paracrystallite" model [17, 18, 19, 20].

The absence of an established model for the structure of a-Si is also due to its structural variability. A variety of pure voidless a-Si's exists [21, 22, 11] with different mechanical 23, 24 and thermodynamic 25] properties depending on preparation method and thermal history. These properties are only well characterized for a small number of experimental a-Si's. The full diversity of pure, voidless a-Si's remains unknown. This lack of fundamental knowledge complicates the assessment of different computational approaches since a model cannot be fully assessed for its validity if the full breadth of experimental structures is not known. In this contribution, we focus on diversity in the SRO and IRO of pure a-Si prepared by different experimental and computational methods and find that order parameters on these length scales vary significantly with material preparation and thermal history ${ }^{3}$

We employ well-established experimental techniques to measure parameters reflecting the SRO and IRO of several forms of pure a-Si. These are ionimplanted and pressure-induced (PI) a-Si [1] and the thermally annealed versions of these materials. The ion-implanted forms have been studied extensively, but the PI a-Si forms have not been assessed in depth. The MRO of these materials has been investigated previously. It appears that while the as-prepared materials differ in structure, thermal annealing structurally relaxes these materials to a common 'lowest-energy' a-Si structure 11]. Such a relaxed network approaches the ideal CRN 21, 22]. It remains unclear how preparation method gives rise to the differences in the as-prepared materials. A previous work attempted to obtain a better understanding of the structure of PI a-Si from a

\footnotetext{
${ }^{3}$ Note that this diversity does not represent a 'polyamorphism', i.e. a transformation between different amorphous polymorphs. Instead, it represents the extensive structural variety within one of these polymorphs, i.e. that of low-density amorphous silicon.
} 
reverse Monte Carlo (RMC) approach constrained by experimental SRO and MRO data [26. The solution space in this approach was large, and there was a significant dependence of the solution on the starting structure. In this work, we develop this understanding further by comparing the as-implanted and relaxed a-Si's to the unknown PI a-Si using vibrational spectroscopy and diffraction.

The structure of a-Si has been studied theoretically through various atomistic modeling methods. The continuous random network (CRN) model for amorphous $\mathrm{SiO}_{2}$ [16] was later extended to a-Si [27, 28]. CRN models are now created through a bond-switching algorithm [29, 30, 31] (often called WWW after Wooten, Winer and Weaire). a-Si models can also be created by the activation-relaxation technique [32] or, more prevalently, molecular dynamics (MD) simulation. In the latter case, the predominant approach is to quench the amorphous network from the melt [33, 34, 35, 36, 37, other options being, e.g. to simulate amorphization through ion implantation [38] or to anneal a pre-created CRN structure using MD [39. Empirical potentials [33, 34, 35, 38] as well as quantum-mechanical tight-binding [39] and ab initio methods [36, 37, 40] have been used to describe the interatomic forces in these simulations. Regardless of method, the resulting models are compared to data obtained from experiment, such as atomic pair-distribution functions [41, 33, 34, 35, 30, 39] or the vibrational density of states [33, 35, 39] in order to validate them. However, it has proven very difficult to create models of a-Si in agreement with all experimental data, and to date no such model exists [42, 43, 44]. In this study, we determine the SRO and IRO characteristics of computer-generated structural models of a-Si constructed using melt-quenching, ion implantation, and a combination of MD and WWW, as well as ad hoc paracrystalline structures, using two different empirical potentials for each case.

We compare the SRO and IRO parameters of the experimental and simulated a-Si structures in depth. The best agreement is found between thermally annealed forms of implanted and PI a-Si and atomistic models prepared using a hybrid WWW and MD approach. Clear qualitative trends are identified in both theory and experiment. Our study reinforces the finding [44, 13] that 
the relaxed state of a-Si approaches the theoretical CRN. The experimental and simulated ion-implanted forms share many characteristics, further evidence that modeling can emulate the complex interplay of melting and quenching during implantation. Finally, analysis of PI a-Si and simulated structures directly quenched from the melt suggests that forms of a-Si created via a mechanism that narrowly avoids crystallization display the highest structural disorder in the short-to-intermediate range among different forms of a-Si.

Our study of experimental and computed a-Si's has broad significance. The magnitude of the band gap in pure a-Si is sensitive to atomic order [45, 46]. Our work demonstrates that SRO/IRO in pure a-Si's may be tuned by preparation method and thermal treatment, showing the possibility of engineering band gaps for specific applications in thin film electronics and optical devices. We identify a strong correlation between preparation method and atomic order, allowing new approaches of predictive modeling of different a-Si's to be developed. Such new methods are critical to correctly predicting the crystallization pathway of a-Si under pressure to one of several metastable crystalline phases. Nanoscale volumes of high-pressure crystalline polymorphs or Si may be highly useful for thin-film technology based on crystallization of a-Si [47, and also for improved photovoltaics [48, 49].

\section{Methods}

\subsection{Experiment}

We experimentally studied four different types of a-Si samples prepared from diamond-cubic (100) Si (dc-Si). These were a-Si amorphized through ion implantation and indentation, and the thermally annealed forms of these two materials.

Continuous $2 \mu \mathrm{m}$ surface layers of implantation-amorphized a-Si were created with the ANU 1.7 MV NEC tandem high energy ion-implanter at liquid nitrogen temperature $(77 \mathrm{~K})$. Silicon ions of 80, 500, 1000, 1500 and $2000 \mathrm{keV}$ in energy were implanted to a fluence of $1 \times 10^{15} \mathrm{~cm}^{-2}$ each into single crystal $\mathrm{Cz}-\mathrm{Si}(100)$, p-doped with boron to a resistivity of $10-20 \Omega \mathrm{cm}$. A tilt of $7^{\circ}$ to the normal 
of the wafer was used for implantation to avoid channeling effects. These films were cut into $3 \mathrm{~mm}$ diameter disks using the Gatan Ultrasonic Disc Cutter 601.

For creating the PI a-Si samples, disks of the same size were first cut from crystalline diamond-cubic $\mathrm{Si}$ (dc-Si). The PI a-Si was then formed using the Ultra-Micro-Indentation System 2000 equipped with a spherical diamond indenter of $\sim 18 \mu \mathrm{m}$ radius by applying loads of up to $750 \mathrm{mN}$ at a loading rate of $5 \mathrm{mN} / \mathrm{s}$ and subsequent fast unloading within $1 \mathrm{~s}$. Some of the implanted and PI samples were then thermally annealed at $450^{\circ} \mathrm{C}$ for 30 minutes in a tube furnace with a nitrogen atmosphere, resulting altogether in four different types of a-Si samples. For ion-implanted a-Si, this heat treatment is well known to structurally relax the film [50] without significant epitaxial regrowth [51], with the same also reported for PI a-Si [1].

These a-Si samples were studied by Raman microspectroscopy to obtain the RMS bond-angle deviation $\Delta \theta$ [52, 21, 53] and a parameter reflecting the dihedral angle deviation $\left(I_{T A} / I_{T O}\right)[54,55,56,15,53$. Raman spectra were recorded with a Renishaw 2000 Raman Imaging Microscope using the $632.8 \mathrm{~nm}$ excitation line of a HeNe laser. Spectra were taken through a $\times 80$ objective using a spot of $\sim 2.0 \mu \mathrm{m}$ radius with a laser power of $\sim 6 \mathrm{~mW}$ focused on the sample, with an accumulation time of $30 \mathrm{~s}$. Under these testing conditions, repeated measurements in the same area confirmed that the samples of a- $\mathrm{Si}$ were not damaged and remained indeed unaltered by the measurement. Further details of the analysis are in Appendix A.

Transmission electron microscopy (TEM) is ideal to study small volumes and thin films. TEM samples were prepared by dimpling to a thickness of $\sim 10 \mu \mathrm{m}$ using a Gatan Dimple Grinder 656 and then further thinning to electron transparency (thickness of $\sim 20 \mathrm{~nm}$ ) by wet-etching using $\mathrm{HNO}_{3}: \mathrm{HF}: \mathrm{CH}_{3} \mathrm{COOH}$ in a mixture of 5:1:1. Electron energy loss spectroscopy (EELS) in the TEM was used to determine the mass density $\rho_{0}$ of each a-Si [57, 58, 59, 60, 61, 62. Differently to previous analysis of the same data using the Richardson-Luczy algorithm [26], we de-convoluted the zero-loss peak from the plasmon peak with the Fourier-Log method [57] for analysis. This became necessary to maintain 
consistency within all four sample sets. We then measured the radial distribution function (RDF) of each form of a-Si in the TEM using high-resolution electron diffraction. The mean coordination number $\langle Z\rangle$, mean bond-angle $\langle\theta\rangle$ as well as $\Delta \theta$ were extracted from the RDF $41,63,64,65,66,67$. This measurement was in large parts consistent with a previous evaluation of the RDF using electron diffraction [26]. The measurement presented here, however, allowed for a larger maximum scattering vector due to splicing together of diffraction patterns [63, 64, 65]. This directly correlates to higher accuracy in real-space data. Full details of these two electron microscopy analyses are presented in Appendix A.

\subsection{Simulation}

In order to gain detailed microscopic insight into the structure of a-Si, we created a set of ensembles of atomistic models of a-Si using computer simulation. Our scope in the present work is understanding SRO and IRO. A future study will focus on MRO in a-Si. Because of this, we required each individual structure to consist of several thousand atoms, thus excluding the use of ab initio methods. Additionally, a thickness of $\sim 20 \mathrm{~nm}$ was imposed on the structures in one dimension, in order to approach TEM sample thickness here and in our future work. We chose to employ the EDIP [68] and Tersoff [69] empirical potentials for the Si-Si interactions in parallel, as both potentials are known to reproduce the experimentally observed decrease in density of Si upon amorphization and to give a reasonable description of the SRO characteristics of the material [38]. Note that the widely used Stillinger-Weber [70] potential does not give the correct trend in density change. Furthermore, the potential displays spurious features in the RDF at short distances [38] and was therefore not employed in this study.

The atomistic ensembles were produced with a range of simulation setups in order to produce a set of different structural models of a-Si. Firstly, to

produce a structural ensemble of $\mathrm{Si}$ amorphized through ion implantation, we performed MD simulations [71, 72, 73, 74, 75, 76, 77] of the amorphization of 
the material using $100 \mathrm{eV}$ self-recoils. The resulting ensemble, created using the EDIP potential, will be referred to as "Implanted EDIP". Secondly, using EDIP and Tersoff in turn, we created an ensemble of a-Si through melting and quenching. These ensembles will be referred to as "Melt-quenched EDIP" and "Melt-quenched Tersoff". Thirdly, for each potential, we produced an ensemble of structures by starting from a set of perfect CRN models and then further relaxing the networks through MD. These ensembles will be referred to as "WWWMD EDIP" and "WWWMD Tersoff". Finally, we created an ensemble with paracrystallites, i.e. highly distorted crystalline grains embedded in an otherwise amorphous structure [17, 18, 19, 20. We did this by substituting spherical crystalline regions into the WWWMD models, and then fully relaxing the systems. These ensembles we term "Paracrystalline EDIP" and "Paracrystalline Tersoff".

To quantify the SRO characteristics of the atomistic models of a-Si, we computed the RDF, $\langle Z\rangle,\langle\theta\rangle$ and $\Delta \theta$ for each ensemble. We also computed number density and difference in potential energy relative to dc-Si. Finally, to access the IRO, we determined the distribution of primitive rings [78] and dihedral angles [40] in each ensemble. Further details of the computational and experimental methods can be found in Appendix B.

\section{Results}

\subsection{Experiment}

\section{Raman microspectroscopy}

Raman spectra for the experimental a-Si's are shown in Fig. 1. For the $2 \mu \mathrm{m}$ thick implanted a-Si, no influence of the substrate is observed. However, in the case of the PI samples, a contribution from the underlying dc-Si can be observed, since the maximum a-Si thickness of $\sim 500 \mathrm{~nm}$ is less than the penetration depth of the laser 4 . The splitting of the transverse optic-like (TO) band is evidence

\footnotetext{
${ }^{4}$ Note that these same dc-Si features are also observed under these indentation conditions independent of unloading rate and thus of phase transformed material formed (i.e. bc $8 / \mathrm{r} 8-\mathrm{Si}$ or PI a-Si). This strongly indicates that they can be attributed to the surrounding matrix of
} 


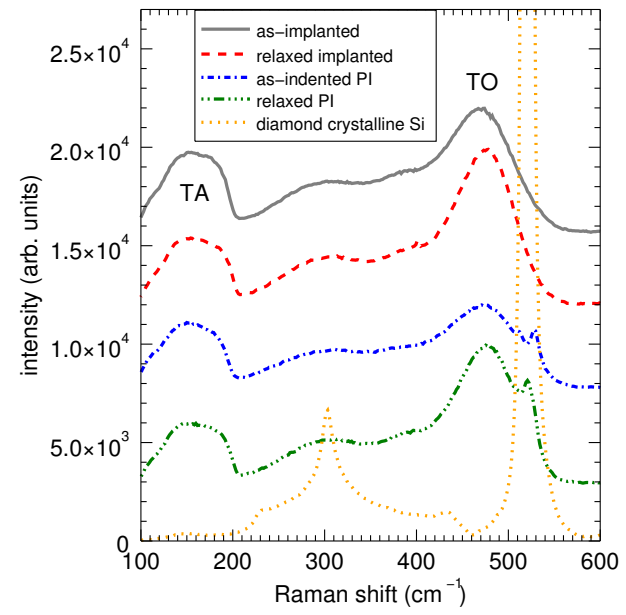

Figure 1: Examples of Raman spectra of as-implanted and as-indented PI as well as relaxed implanted and relaxed PI a-Si with a dc-Si spectrum as background. The amorphous spectra are all offset for clarity.

of residual stress in the indented specimens [79, 80, 81, 82,

The averaged SRO/IRO parameters obtained from five Raman spectra for each a-Si are presented with their standard errors in Table 1 . The quantitative measures of $\Delta \theta$ and $I_{T A} / I_{T O}$ show significant differences between samples created through the four different preparation methods. For both implanted and $\mathrm{PI}$ a-Si, thermal annealing of the samples leads to a more ordered network, as reflected in the change in both $\Delta \theta$ and $I_{T A} / I_{T O}$ with respect to their as-prepared forms.

\section{Electron microscopy}

The averaged values of plasmon peak energy $E_{p}$ and $\rho_{0}$ determined from five EELS spectra from five different areas of each a-Si are presented in Table $2 \rho_{0}$ of as-prepared and relaxed ion-implanted a-Si determined by volumetric analysis are $2.287 \pm 0.003 \mathrm{~g} / \mathrm{cm}^{3}$ and $2.289 \pm 0.002 \mathrm{~g} / \mathrm{cm}^{3}$, respectively [83], in excellent

dc-Si and not the phase transformed material. Furthermore, extensive transmission electron microscopy of such phase transformed zones demonstrates the sole presence of amorphous material. 


\begin{tabular}{c||c|c|c} 
Specimen & $\begin{array}{c}\Gamma_{T O} / 2 \\
{\left[\mathrm{~cm}^{-1}\right]}\end{array}$ & $\begin{array}{c}\Delta \theta \\
{\left[{ }^{\circ}\right]}\end{array}$ & $I_{T A} / I_{T O}$ \\
\hline \hline As-Implanted & $39.8 \pm 0.4$ & $10.8 \pm 0.1$ & $0.636 \pm 0.019$ \\
Relaxed Implanted & $34.7 \pm 0.3$ & $9.1 \pm 0.1$ & $0.395 \pm 0.006$ \\
As-Indented PI & $42.0 \pm 0.2$ & $11.5 \pm 0.1$ & $0.744 \pm 0.008$ \\
Relaxed PI & $36.1 \pm 0.1$ & $9.5 \pm 0.1$ & $0.432 \pm 0.002$
\end{tabular}

Table 1: Results extracted from the measured Raman spectra.

\begin{tabular}{c||c|c|c|c|c|c} 
Specimen & $E_{P}(\mathrm{eV})$ & $\rho_{0}\left(\mathrm{gcm}^{-3}\right)$ & $\rho_{0} / \rho_{\text {crystal }}$ & $\langle Z\rangle$ & $\langle\theta\rangle\left(^{\circ}\right)$ & $\Delta \theta\left(^{\circ}\right)$ \\
\hline \hline As-Implanted & $16.61 \pm 0.06$ & $2.287 \pm 0.008$ & $0.982 \pm 0.003$ & $3.7 \pm 0.3$ & $110.0 \pm 1.0$ & $10.2 \pm 0.1$ \\
Relaxed Implanted & $16.62 \pm 0.08$ & $2.290 \pm 0.012$ & $0.984 \pm 0.005$ & $3.9 \pm 0.3$ & $111.0 \pm 0.6$ & $9.6 \pm 0.2$ \\
As-Indented PI & $16.76 \pm 0.07$ & $2.328 \pm 0.010$ & $1.000 \pm 0.004$ & $3.6 \pm 0.1$ & $109.0 \pm 0.6$ & $11 \pm 1$ \\
Relaxed PI & $16.56 \pm 0.05$ & $2.274 \pm 0.007$ & $0.977 \pm 0.003$ & $3.9 \pm 0.1$ & $110 \pm 1$ & $8.4 \pm 0.5$
\end{tabular}

Table 2: Density and SRO parameters extracted from the measured EELS spectra and $J(r)$ s, respectively.

agreement with our measurements. Interestingly, for as-indented PI a-Si, $\rho_{0}$ approaches that of dc-Si within experimental error. This elevated mass density correlated well with measurements of mechanical hardness $11,84,5$. The originally high density of PI a-Si decreases significantly upon annealing, in contrast to what is observed for implanted a-Si, where the density is unchanged within experimental error upon the relaxation. The $\rho_{0}$ of relaxed PI a-Si is the same, within error, as that of relaxed ion-implanted a-Si. This is in accordance with expectations, as relaxed PI and relaxed ion-implanted a-Si have previously been found to share many structural characteristics [11.

Representative reduced RDFs from the four different a-Si specimens are displayed in Fig. 2. The SRO parameters of the networks extracted from these experimental RDFs are displayed in Table 2. The measured coordination numbers $\langle Z\rangle$ for the as-implanted and relaxed implanted samples of $3.7 \pm 0.3$ and $3.9 \pm 0.3$, respectively, are in good agreement with those extracted from high-resolution X-ray diffraction data, which were $3.79 \pm 0.01$ and $3.88 \pm 0.01$, respectively [22].

\footnotetext{
${ }^{5}$ Note that despite its elevated density PI a-Si remains a 'low-density' polymorph as evidenced by its coordination below 4 rather than a 'high-density' polymorph with a coordination above 4.
} 
This increase in $\langle Z\rangle$ upon thermal annealing is indicative of short-range ordering from structural relaxation and has been attributed to defect annihilation [21, 22] based on Raman spectroscopy and differential scanning calorimetry. The asindented PI stands out as having the lowest $\langle Z\rangle$ of the four samples, consistent with a previous RDF electron diffraction study on this material that did not use such a large range of scattering vector [84. Thermal annealing of the PI a-Si, however, results in structural relaxation reflected in the increase in $\langle Z\rangle$ to the same level as in relaxed implanted a-Si. The bond angle deviation, $\Delta \theta$, decreases upon thermal annealing for both the ion-implanted and PI a-Si, in accordance with the structural relaxation inferred from the change in $\langle Z\rangle$ upon the heat treatment. We plot the bond-angle deviation parameters from Raman analysis and the RDF for comparison in Fig. 6(a).

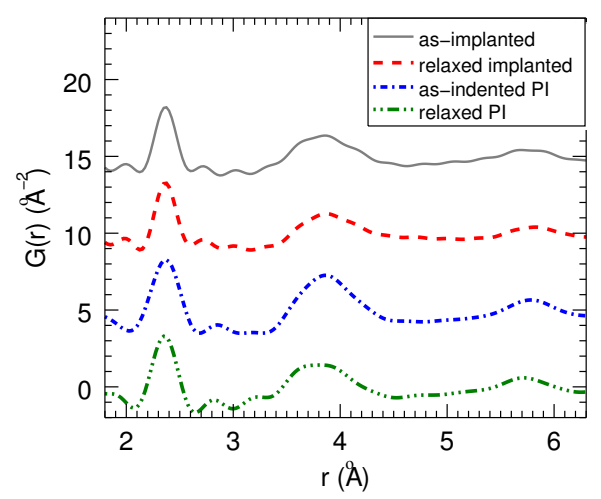

Figure 2: Experimental reduced RDFs for the studied four forms of a-Si. The upper three $G(r)$ curves have been offset by $5 \AA^{-2}$ for clarity.

\subsection{Simulation}

By comparing the SRO characteristics and the reduced RDFs of the computergenerated structural ensembles with the present experimental results, it was found that only six of the created ensembles showed satisfactory agreement with experiment. The general features of $G(r)$ for these ensembles (Fig. 3) are in good agreement with the experimental RDFs (Fig. 2). However, the computed $G(r)$ have a tendency to display a very small peak at $3 \AA$ not present in 
the experimental $G(r)$. This arises from the inherent cutoffs in the potentials used and highlights the fundamental difficulty in exact replication of experimental data with computation. These small peaks do not, however, affect any consequent analysis or conclusions drawn.

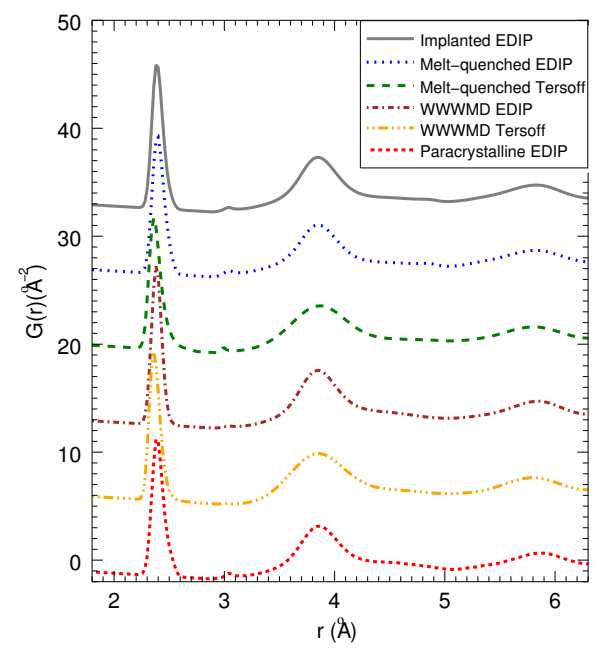

Figure 3: Reduced RDFs for the simulated materials. The upper $G(r)$ curves have been offset by $7 \AA^{-2}$ for clarity.

The bulk properties and coordination characteristics for the same six computergenerated ensembles are presented in Table 3. The structures are found to be energetically stable, as quantified by a mean potential energy $E-E_{c}$ higher than that of dc-Si by $\sim 0.25 \mathrm{eV} /$ atom. All ensembles show the correct trend of decreased density with respect to dc-Si, with the density relative to dc-Si being broadly similar to the corresponding result from experiment $\left(\rho_{0} / \rho_{\text {crystal }} \approx\right.$ 0.98), save for the high-density as-indented PI sample. While the distribution of coordination numbers $\langle Z\rangle$ will be discussed below, we note here that the atomistic models tend to be overcoordinated with respect to the experimental samples of a-Si [85]. The mean bond-angle $\langle\theta\rangle$ is systematically $\sim 1^{\circ}$ lower in simulation than in experiment, whereas $\Delta \theta$ tends to be slightly higher.

By examining $\Delta \theta$ in more detail, it is seen that the WWWMD structures display clearly the lowest bond-angle deviation among the ensembles. To ex- 


\begin{tabular}{|c|c|c|c|c|c|c|c|c|c|}
\hline Name of ensemble & $\| N_{\text {atoms }}$ & Dimensions (nm) & $E-E_{c}(\mathrm{eV})$ & $\rho_{0} / \rho_{\text {crystal }}$ & $\mathrm{Z}=3$ & $\mathrm{Z}=4$ & $\mathrm{Z}=5$ & $\mathrm{Z}=6$ & $\langle Z\rangle$ \\
\hline ited EDIP & 65536 & $8.8 \times 8.8 \times 18$ & 0.24 & 0.964 & 0.01 & 0.88 & 0.10 & 0.0 & 4.1 \\
\hline enched EDIP & 65536 & $8.8 \times 8.8 \times 18$ & 0.26 & 0.973 & 0.01 & 0.83 & 0.15 & 0.0 & 4.1 \\
\hline Melt-quenched Tersoff & 65536 & $8.8 \times 8.8 \times 18$ & 0.29 & 0.975 & 0.04 & 0.85 & 0.11 & 0.0 & 4.1 \\
\hline WWWMD EDIP & 7200 & $2.7 \times 2.7 \times 19$ & 0.20 & 0.972 & 0.0 & 0.99 & 0.01 & 0.0 & 4.0 \\
\hline WWWMD Tersoff & 7200 & $2.7 \times 2.7 \times 20$ & 0.23 & 0.978 & 0.0 & 0.99 & 0.01 & 0.0 & 4.0 \\
\hline Paracrystalline EDIP & 7233 & $2.8 \times 2.8 \times 20$ & 0.27 & 0.966 & 0.03 & 0.85 & 0.12 & 0.0 & 4.1 \\
\hline
\end{tabular}

Table 3: SRO and bulk characteristics of those computer-generated structural ensembles that showed satisfactory agreement with experiment. $N_{\text {atoms }}$ stands for the average number of atoms per structure in each ensemble. $Z=i$ signifies the fraction of atoms in the ensemble with a first-shell coordination of $i$.

\begin{tabular}{c||c|c|c|c|c} 
Name of ensemble & $R=3$ & $R=4$ & $R=5$ & $R=6$ & $R=7$ \\
\hline \hline Implanted EDIP & 0.03 & 0.08 & 0.51 & 0.68 & 0.46 \\
Melt-quenched EDIP & 0.05 & 0.11 & 0.54 & 0.65 & 0.46 \\
Melt-quenched Tersoff & 0.01 & 0.08 & 0.51 & 0.73 & 0.48 \\
WWWMD EDIP & 0.0 & 0.02 & 0.42 & 0.83 & 0.56 \\
WWWMD Tersoff & 0.0 & 0.01 & 0.42 & 0.83 & 0.56 \\
Paracrystalline EDIP & 0.08 & 0.06 & 0.40 & 0.78 & 0.49 \\
\hline dc-Si & 0.0 & 0.0 & 0.0 & 2.00 & 0.0 \\
\hline
\end{tabular}

Table 4: Distribution of primitive rings (see [78]) in each ensemble as given by the total number of rings per total number of atoms. Statistics for standard dc-Si are included for comparison. $R$ stands for the size of the ring.

plain this, we refer to the full bond-angle distributions of the atomistic models (Fig. 4). Here we find the main peak at around $109^{\circ}$ to be accompanied by two smaller peaks, around $60^{\circ}$ and $75^{\circ}$, for Melt-quenched EDIP, Implanted EDIP, and Paracrystalline EDIP, with Melt-quenched Tersoff displaying only the peak at $75^{\circ}$. By studying the ring statistics for the ensembles 38 (Table 4), it is apparent that the peak $\sim 60^{\circ}$ is due to three-membered rings and the peak $\sim 75^{\circ}$ is due to four-membered rings. The existence of these two auxiliary peaks in the bond-angle distribution increases $\Delta \theta$ for all the ensembles except WWWMD. The trends in $\Delta \theta$ (visualized in Fig. 6(b)) therefore reduce to trends in the relative abundance of small primitive rings among the ensembles.

The distribution of dihedral angles is presented in Fig. 5 for each ensemble. For a perfect lattice of dc-Si, the distribution would show two sharp peaks at $60^{\circ}$ and $180^{\circ}$, respectively. For the amorphous ensembles, the spread in $\Delta \theta$ is generally matched with a corresponding spread in dihedral angles around the positions of $60^{\circ}$ and $180^{\circ}$, with the WWWMD structures showing sharper 


\begin{tabular}{c||c|c|c} 
Name of ensemble & $\langle\theta\rangle\left(^{\circ}\right)$ & $\Delta \theta\left(^{\circ}\right)$ & $1000 / P_{\max }^{\varphi}$ \\
\hline \hline Implanted EDIP & 108.3 & 15.9 & 0.464 \\
Melt-quenched EDIP & 107.9 & 17.6 & 0.656 \\
Melt-quenched Tersoff & 108.7 & 14.6 & 0.369 \\
WWWMD EDIP & 109.0 & 12.0 & 0.246 \\
WWWMD Tersoff & 109.2 & 11.0 & 0.232 \\
Paracrystalline EDIP & 107.7 & 17.4 & 0.304 \\
\hline
\end{tabular}

Table 5: SRO and IRO characteristics of the computer-generated structural ensembles as expressed by the mean bond angle, the bond angle distortion, and the spread of the dihedral angle distributions (see text).

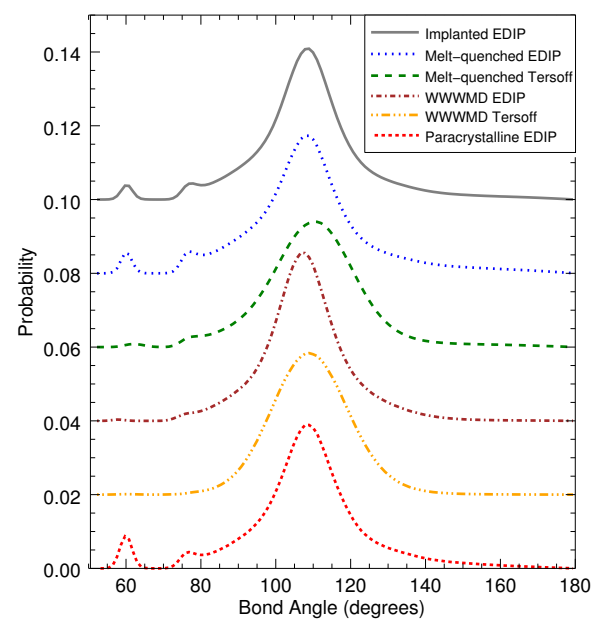

Figure 4: Bond-angle distribution for each structural ensemble.

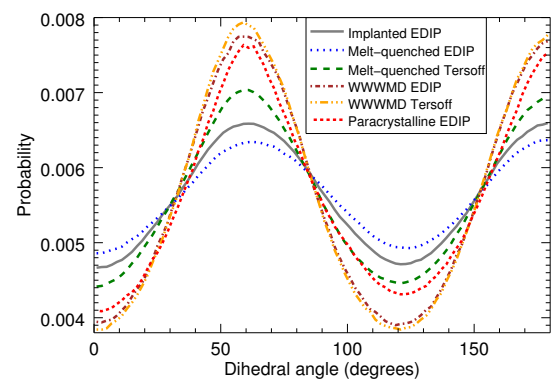

Figure 5: Dihedral angle distribution for each structural ensemble. 
distributions than the implanted and melt-quenched ensembles. The paracrystalline model shows mixed behavior with a high $\Delta \theta$ coupled to a dihedral angle distribution more strongly peaked than the melt-quenched or implanted models. We use the maximum in the probability distribution (Fig. 5) of dihedral angles $\left(P_{\max }^{\varphi}\right)$ to quantify this trend. To enable qualitative comparison to the experimentally accessed IRO, it is useful to consider the inverse, $1 / P_{\max }^{\varphi}$. For a perfect lattice of dc-Si the probability becomes a two-fold, sharp delta function yielding for $1 / P_{\max }^{\varphi}$ a value of zero. This is clearly consistent with the experimental IRO where $I_{T A} / I_{T O}$ also equals zero. The computed IRO results of the structural models are summarized in Table 5, and the comparison to the experimental counterpart, $I_{T A} / I_{T O}$, is shown in Figs 6 (c) and (d). Note that to ease comparison and to display the experimental ratio and simulated $1 / P_{\max }^{\varphi}$ on the same scale, $1000 / P_{\max }^{\varphi}$ is used in the table and figure.

The SRO and IRO characteristics expressed by the bond-angle and dihedral angle distributions are summarized in Table 5. For the fully amorphous ensembles, i.e. excluding the paracrystalline ensemble, the WWWMD networks display the highest IRO, followed by the implanted and then melt-quenched ones. These findings are thus fully consistent with trends observed from bondangle distributions (Table 5 and Figs 6 (b) and (d)).

\section{Discussion}

\subsection{Experiment}

Strong correlation in the qualitative trends is found between Raman and RDF data (Fig. 6 (a)). The lack of quantitative agreement is not surprising given that the formula to extract $\Delta \theta$ from the Raman spectra is empirically derived [52. The reduction in $\Delta \theta$ of as-implanted a-Si upon thermal annealing is in agreement with previous studies [21, 11]. $\Delta \theta$ of both relaxed forms of a-Si approach a similar value. As-indented $\mathrm{PI}$ a-Si exhibits a higher $\Delta \theta$ than the other forms of a-Si, indicating less structural order on the SRO scale in this network. Furthermore, the structural characteristics of the IRO confirm this finding, since as-indented PI a-Si also exhibits the least order on this length 
Experiment
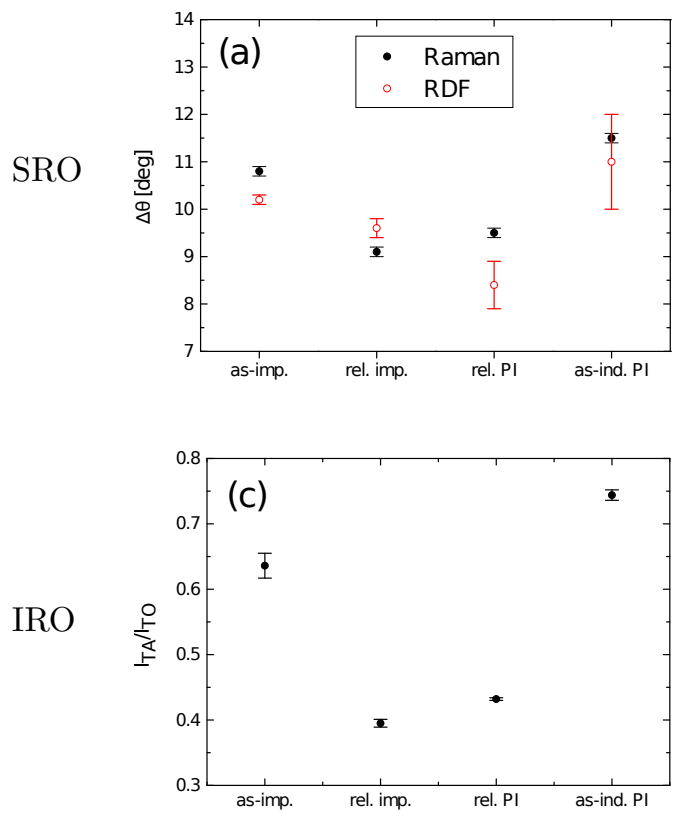

Simulation
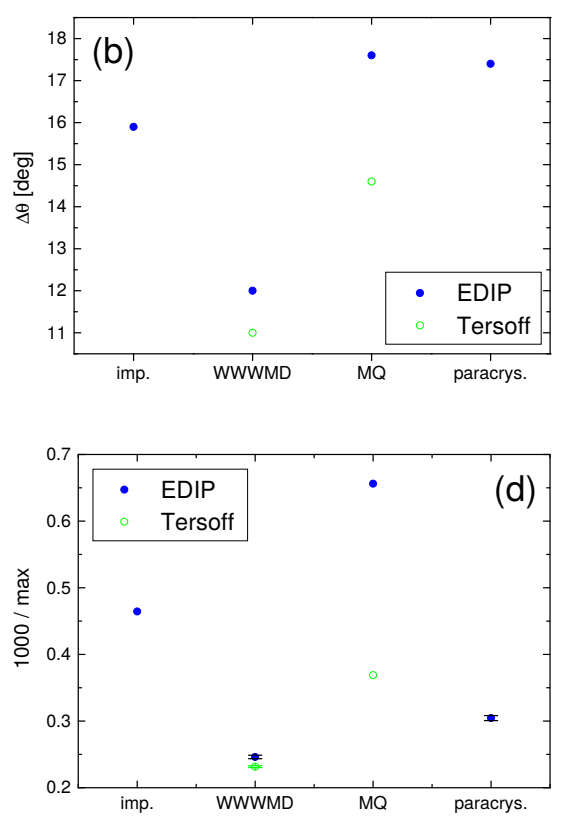

Figure 6: Top row: Comparison of the SRO parameter $\Delta \theta$ from (a) experiment and (b) simulation. Note that the same total range of $7^{\circ}$ was chosen on the $y$-axis in (a) and (b) to ease comparison of the two. Bottom row: Comparison of (c) the experimental IRO parameter $I_{T A} / I_{T O}$ and $(\mathrm{d})$ the IRO parameter $\left(1 / P_{\max }^{\varphi} \times 1000\right)$ characterizing the dihedral angle distribution of each simulated ensemble (see text). Note that the same total range of 0.5 was chosen on the y-axis in (c) and (d) to ease comparison. In all figures, black solid circles are from Raman spectroscopy, red open circles are derived from the experimental RDFs, blue solid circles are from simulations using EDIP, and green open circles are from those using Tersoff. 
scale (Table 1. Fig. 6(c)). In fact, the degrees of SRO and IRO appear strongly correlated in all experimental a-Si's, as seen in the similar shapes of Figs 6 (a) and (c). This is true also for the simulated forms of a-Si, as seen in Figs 6 (b) and $(d)$.

In a completely random arrangement of atoms, an increase in mass density is accompanied by a linear increase in mean coordination number for a fixed cutoff distance. Amorphous materials have significant atomic short-range ordering, giving rise to strong features in the RDF such as the first-nearest neighbour peak. Even so, experimentally, the nearest-neighbour co-ordination shell is not well defined, as the RDF may not exactly go to zero. From the experimental RDFs, we measure $\langle Z\rangle$ as integrated counts over a constant range of $r=2.1$ to $3.0 \AA$ for all measurements as the first-nearest neighbour peak is not well-defined enough to motivate changing the co-ordination shell radius from specimen to specimen [63. In our implanted a-Si's, the density remains unchanged, even though measured coordination in this fixed shell increases upon thermal relaxation. This indicates that short-range ordering is occurring during the relaxation anneal, resulting in a sharper first-neighbour peak in the RDF. For the PI a-Si's, we observe a decrease in density and increase in coordination upon relaxation. This trend demonstrates that in the as-indented material there is significant disorder in the first-nearest neighbour shell, and a spreading of nearest neighbour distances beyond the fixed cut-off used to measure the coordination.

Higher-density crystalline polymorphs of Si $(\beta$-Sn, Si-II [86, 87, 88, 89, SiXII (r8), and Si-III (bc8) [90, 91, 92, 93]) are formed from dc-Si via high pressure in a diamond-anvil cell or via indentation. PI a-Si is formed when an indenter is unloaded quickly and the nucleation of the bc8 and r8 structures is avoided 92 , 11. The rate-dependence of amorphization in this "pressure-quench" is thus somewhat similar to when quenching from the melt. The as-indented PI a-Si is the densest form of a-Si measured to date, reflecting the method of preparation. 


\subsection{Simulation}

Structural diversity also exists for the simulated forms of a-Si. We now explore the variety introduced into our computer-generated structures of a-Si by the choice of interatomic potential and generation method.

Two categories of ensembles, WWWMD and melt-quenched, were prepared using both the Tersoff and EDIP potentials. The materials prepared using Tersoff show consistently higher energies, lower $\Delta \theta$ (Fig.6(b)), a higher fraction of undercoordinated and tetrahedrally coordinated atoms, and a larger number of six-membered rings. The difference in $\Delta \theta$ is larger between the melt-quenched than the WWW ensembles prepared using the two potentials. This difference is also pronounced in the IRO (Fig. 6(d)). This effect may be due to differences in the original melt. While $\langle Z\rangle$ of the liquid at $2000 \mathrm{~K}$ varies only slightly between the two potentials (4.3 for Tersoff, 4.5 for EDIP), the density of the Tersoff liquid is significantly higher than that of dc-Si, whereas the EDIP liquid is less dense 68.

In order to probe the effect of generation method on structure, we compare the four different ensembles prepared using the EDIP potential, starting from coordination statistics. The WWWMD displays almost perfect fourfold coordination, as expected. The implanted ensemble and the melt-quenched ensemble have slight overcoordination through an increase in fivefold-coordinated atoms. The overcoordination in simulated melt-quenched and implanted a-Si has been documented previously [38, and remains an unresolved problem for these empirical potentials. The paracrystalline structure has a fraction of $Z=5$ atoms intermediate between the melt-quenched and implanted ensembles and a finite amount of $Z=3$ atoms. These differences in coordination are reflected in the ring statistics for the ensembles (Table 4). For dc-Si, the only ring size present in the size range considered here is $R=6$. The WWWMD structures are almost solely made up of five, six, and seven-membered rings, still giving $\langle Z\rangle=4.0$. However, the other EDIP ensembles display a shift in ring distributions towards smaller ring sizes, implying overcoordination as observed above. 


\subsection{Correlation between experimental and simulated structures}

Overall, the strong structural variability of computer-generated a-Si with preparation method and thermal history mirrors our observations of the experimental materials, and trends can be identified. However, quantitative agreement between simulation and experiment cannot always be expected. For example, in simulation, the self-consistent way to find $\langle Z\rangle$ is to integrate the pair-distribution function up to the first minimum beyond the first peak. In experiment, we measure $\langle Z\rangle$ as integrated counts over a constant range of $r=2.1$ to $3.0 \AA$ for all measurements in order to avoid termination ripples at low $r[63$. The difference in integration range between the two methods may constitute an additional source of discrepancy between simulation and experiment.

Experimentally relaxed forms of a-Si and the WWWMD structures

The thermally relaxed a-Si's and the WWWMD models are strikingly similar, with the highest SRO and IRO with respect to other experimental or theoretical generation methods, almost perfect fourfold-coordination, and a similar density deficit with respect to dc-Si. Our findings imply that the WWW bondswitching algorithm is a similar process to structural relaxation in experiment. Indeed, it has been long argued that relaxed a-Si approaches the experimental equivalent of a CRN [11, 21, 22, 44, 13.

Experimentally as-implanted $a-S i$ and simulated implanted a-Si

The experimental as-implanted a-Si and the simulated implanted a-Si do not compare quantitatively, although they share the same mechanism of amorphization. The simulated implanted a-Si is slightly overcoordinated and less dense than the experimental material. These observations reiterate previous findings that implantation-amorphized Si cannot be completely accurately produced using standard classical interaction potentials and MD simulation [38. Whereas the temperature-quenching of liquid-like collision cascades is captured realistically in standard MD simulation, the time between successive recoil events is orders of magnitude shorter than in typical experiments. The simulation is 
therefore likely to neglect relaxation of defect configurations (including recombination of close defects) with relaxation barriers higher than those that can be overcome within short (ps) time scales, contributing to discrepancies between theory and experiment. Nonetheless, the implanted a-Si's compare qualitatively, as they are the second-most ordered a-Si's, midway between the relaxed and quenched forms (Fig. 6).

Pressure-induced a-Si and simulated melt-quenched a-Si

PI a-Si and melt-quenched a-Si's are the least ordered of all forms considered experimentally and computationally. Both are formed through quench rates that are just sufficiently fast enough to avoid crystallization. In the melt-quenched case this means a cooling rate of $0.01 \mathrm{~K} / \mathrm{fs}$ or $10^{13} \mathrm{~K} / \mathrm{s}$, which prohibits nucleation of dc-Si during cooling. In the case of the PI a-Si, the analogue of rapid quenching is the rapid pressure-release rate of $\sim 10 \mathrm{GPa} / \mathrm{s}$, which is fast enough to prevent nucleation of the crystalline bc 8 and $r 8$ structures. The a-Si's do not compare quantitatively, similar to the implanted a-Si's, as these processes are sensitive to rate, and the simulation could not match the experimental time scales.

The paracrystalline ensemble

Unlike the other models, the paracrystalline model has no experimental equivalent in this study. The low degree of SRO and high degree of IRO (Figs 6 (b) and (d)) is not consistent with any known experimental form of a-Si, where high IRO always corresponds to high SRO and vice versa.

\subsection{Significance of variation in structural order}

Through our combined experimental and modeling study we have been able to distinguish clear trends in structural order of a-Si with preparation technique. Our work provides an initial guide for engineering desirable properties in this elemental amorphous system, and also for following a new approach of predictive modeling of a-Si.

A low defect density is critical in silicon photonics based on a-Si [94. Previous experiments [46] have demonstrated that the magnitude of the band gap 
in nominally pure a-Si's is extremely sensitive to preparation method, varying from $0.95 \mathrm{eV}$ for sputtered a-Si to $1.3 \mathrm{eV}$ for a-Si prepared using electron-beam evaporation. Modeling studies also show a variation in the band gap as a function of preparation method, with the WWW model having a larger band gap and fewer gap states than a model prepared by melt-quenching [45]. Our study demonstrates that order in the short-intermediate range can be varied strongly by controlling preparation method, with a smaller band gap anticipated for generation methods yielding higher disorder [45, 95]. Our work shows that a significant amount of electronic structure engineering can be achieved even for these elemental amorphous semiconductors, which are used widely in thin-film transistor and solar cell technology.

Phase transformation to the bc8/r8 Si polymorphs can be induced by indentation pressure in a-Si enabling impurity-free crystallization of a-Si at room temperature [47, an alternative to more expensive laser-crystallization [96]. These localized phase transformations can be exploited for maskless nanoscale patterning simply through the differing etch rates of these different Si phases [97. The bc8 and $\mathrm{r} 8$ polymorphs are themselves of interest, with both phases predicted to be significantly better suited for photovoltaic applications than standard dc$\mathrm{Si}$ or a-Si [48, 49]. The correlations we have found between experimental and modeled structures and the demonstrated universal dependence of structure on preparation method may enable new avenues for engineering such nanoscale patterning. To this end, we propose an approach of predictive modeling where a form of a-Si, sufficiently rigid to undergo phase transformation instead of plastic flow [98, is first created in simulation, and then this preparation method is emulated is experiment, to enable following a particular crystallization pathway to a desirable high-pressure metastable phase.

\section{Conclusions}

Large variation in atomic ordering is found between experimental and simulated forms of a-Si, regardless of their voidless, pure, and uniform nature. The only simulated a-Si that attained quantitative agreement with experiment 
was that generated through a hybrid bond-switching Monte Carlo and MD approach, giving a structure very close to the experimentally thermally relaxed a-Si's. Simulation methods that depend on rate, such as ion-implantation and melt-quenching, could not match the measured SRO/IRO parameters, as the simulated time scales are too short. Nonetheless, clear relative differences were identified in SRO/IRO in both theory and experiment. a-Si quenched from extreme conditions is the most disordered. a-Si formed from implantation is more ordered, and relaxed forms of a-Si are the most ordered, regardless of preparation method preceding the relaxation. No unique experimental form and therefore no unique structural model exists for a-Si. By mimicking experimental setups in simulation, qualitative agreement in structural properties can be achieved. Our work serves to guide engineering a-Si for desireable properties in existing photonics, and our findings enable a new approach of predictive modeling of a-Si for future applications.

\section{Acknowledgements}

BH gratefully acknowledges funding from an Alvin M. Weinberg Fellowship (ORNL) and through the Spallation Neutron Source, sponsored by the U.S. Department of Energy, Office of Basic Energy Sciences. ACYL wishes to acknowledge the support of the Margaret Clayton Women In Research Fellowship, the Science Faculty, Monash University and the Monash Centre for Electron Microscopy. BH and ACYL thank Vicki Keast for discussions on treatment of low-loss EELS spectra and Nestor Zaluzec for assistance with initial electron diffraction measurements at Argonne National Laboratory. JEB acknowledges funding from the Australian Research Council Future Fellowship scheme. The authors acknowledge use of facilities within the Monash Centre for Electron Microscopy and the Electron Microscopy Center, Argonne National Laboratory. OHP gratefully acknowledges funding from the Academy of Finland and grants of computation time from CSC - IT Center for Science Ltd (Finland). EH gratefully acknowledges generous grants of computing time on the Ametisti cluster at the University of Helsinki and the Vuori HP CP4000 BL ProLiant supercluster 
at CSC. We acknowledge financial support by the Academy of Finland through the Centres of Excellence Program (Project No. 251748).

[1] T. Vogt, T. Shinbrot, Overlooking glass?, Phys. Rev. Applied Editorial (May 19th 2015).

[2] M. J. Powell, The physics of amorphous-silicon thin-film transistors, IEEE Transactions on Electron Devices 36 (1989) 2753-2763.

[3] D. E. Carlson, C. R. Wronski, Amorphous silicon solar cells, Applied Physics Letters 28 (1976) 671-374.

[4] J. Robertson, Properties of diamond-like carbon, Surface and Coatings Technology 50 (1992) 185-203.

[5] Y. Lifshitz, Diamond-like carbon - present status, Diamond and Related Materials 8 (1999) 1659-1676.

[6] R. C. Powles, N. A. Marks, D. M. Lau, D. G. McCulloch, D. R. McKenzie, An energy landscape for carbon network solids, Carbon 63 (2013) 416.

[7] M. Wuttig, N. Yamada, Phase-change materials for rewriteable data storage, Nature Materials 6 (2007) 824-832.

[8] L. Stixrude, B. Karki, Structure and freezing of mgsio3 liquid in earth's lower mantle, Science 310 (2005) 297.

[9] B. Karki, D. Bhattarai, L. Stixrude, First-principles simulations of liquid silica: Structural and dynamical behavior at high pressure, Phys. Rev. B 76 (2007) 104205.

[10] S. Klotz, G. Hamel, J. S. Loveday, R. J. Nelmes, M. Guthrie, A. K. Soper, Structure of high-density amorphous ice under pressure, Phys. Rev. Lett. 89 (2002) 285502.

[11] B. Haberl, A. C. Y. Liu, J. E. Bradby, S. Ruffell, J. S. Williams, P. Munroe, Structural characterization of pressure-induced amorphous silicon, Physical Review B 79 (2009) 155209. 
[12] M. M. J. Treacy, K. B. Borisenko, The local structure of amorphous silicon, Science 335 (2012) 950.

[13] S. Roorda, L. J. Lewis, Comment on 'the local structure of amorphous silicon', Science 338 (2012) 1539.

[14] J. M. Ziman, Models of Disorder: The Theoretical Physics of Homogeneously Disordered Systems, Cambridge University Press, Cambridge, 1979.

[15] S. Gupta, R. S. Katiyar, G. Morell, S. Z. Weisz, I. Balberg, The effect of hydrogen on the network disorder in hydrogenated amorphous silicon, Applied Physics Letters 75 (1999) 2803-2805.

[16] W. H. Zachariasen, The atomic arrangement in glass, Journal of the American Chemical Society 54 (1932) 3841-3851.

[17] M. M. J. Treacy, K. B. Borisenko, The local structure of amorphous silicon, Science 335 (2012) 950.

[18] S. N. Bogle, P. M. Voyles, S. V. Khare, J. R. Abelson, Quantifying nanoscale order in amorphous materials: simulating fluctuation electron microscopy of amorphous silicon, J. Phys.: Condens. Matter 19 (2007) 455204 .

[19] J. M. Gibson, M. M. J. Treacy, Diminished medium-range order observed in annealed amorphous germanium, Physical Review Letters 78 (1997) 1074-1077.

[20] K. Nordlund, J. Keinonen, E. Rauhala, T. Ahlgren, Range profile in self-ion-implanted crystalline si, Phys. Rev. B 52 (1995) 15170.

[21] S. Roorda, W. C. Sinke, J. M. Poate, D. C. Jacobson, S. Dierker, B. S. Dennis, D. J. Eaglesham, F. Spaepen, P. Fuoss, Structural relaxation and defect annihilation in pure amorphous silion, Physical Review B 44 (1991) $3702-3725$. 
[22] K. Laaziri, S. Kycia, S. Roorda, M. Chicoine, J. L. Robertson, J. Wang, S. C. Moss, High-energy X-ray diffraction study of pure amorphous silicon, Physical Review B 60 (1999) 13520-13533.

[23] B. Haberl, J. E. Bradby, S. Ruffell, J. S. Williams, P. Munroe, Phase transformations induced by spherical indentation in ion-implanted amorphous silicon, Journal of Applied Physics 100 (2006) 013520-1-013520-9.

[24] B. Haberl, J. E. Bradby, M. V. Swain, J. S. Williams, P. Munroe, Phase transformations induced in relaxed amorphous silicon by indentation at room temperature, Applied Physics Letters 85 (2004) 5559-5561.

[25] M. G. Grimaldi, P. Baeri, M. A. Malvezzi, Phys. Rev. B 44 (1991) 1546.

[26] K. B. Borisenko, B. Haberl, A. C. Y. Liu, Y. Chen, G. Li, J. S. Williams, J. E. Bradby, D. J. H. Cockayne, M. M. J. Treacy, Medium-range order in amorphous silicon investigated by constrained structural relaxation of two-body and four-body electron diffraction data, Acta Materialia 60 (2012) 359375.

[27] D. E. Polk, Structural model for amorphous silicon and germanium, Journal of Non-Crystalline Solids 5 (1971) 365-376.

[28] D. Beeman, B. L. Bobbs, Computer restructuring of continuous random tetrahedral networks, Physical Review B 12 (1975) 1399-1403.

[29] F. Wooten, K. Winer, D. Weaire, Phys. Rev. Lett. 54 (1985) 1392.

[30] G. T. Barkema, N. Mousseau, High-quality continuous random networks, Physical Review B 62 (2000) 4985-4990.

[31] S. von Alfthan, A. Kuronen, K. Kaski, Realistic models of amorphous silica: A comparative study of different potentials, Phys. Rev. B 68 (2003) 073203.

[32] G. T. Barkema, N. Mousseau, Event-based relaxation of continuous disordered systems, Physical Review Letters 77 (1996) 4358-4361. 
[33] R. Biswas, G. S. Grest, C. M. Soukoulis, Generation of amorphous-silicon structures with use of molecular-dynamics simulations, Physical Review B 36 (1987) 7437-7441.

[34] M. D. Kluge, J. R. Ray, A. Rahman, Amorphous silicon formation by rapid quenching: A molecular-dynamics study, Physical Review B 36 (1987) 4234-4237.

[35] W. D. Luedtke, U. Landman, Preparation, structure, dynamics, and energetics of amorphous silicon: A molecular-dynamics study, Physical Review B 40 (1989) 1164-1174.

[36] R. Car, M. Parrinello, Structural, dynamical, and electronic properties of amorphous silicon: An ab initio molecular-dynamics study, Phys. Rev. Lett. 60 (1988) 204.

[37] D. A. Drabold, P. A. Fedders, O. F. Sankey, J. D. Dow, Moleculardynamics simulation of amorphous si, Phys. Rev. B 42 (1990) 5135.

[38] J. Nord, K. Nordlund, J. Keinonen, Phys. Rev. B 65 (2002) 165329.

[39] N. Bernstein, J. L. Feldman, M. Fornari, Structural model of amorphous silicon annealed with tight binding, Physical Review B 74 (2006) 205202/1-10.

[40] I. Stich, R. Car, M. Parrinello, Phys. Rev. B 44 (1991) 11092.

[41] D. J. H. Cockayne, The study of nanovolumes of amorphous materials using electron scattering, Annual Review of Materials Research 37 (2007) 159-187.

[42] D. Drabold, J. Li, Approximate ab initio simulations of amorphous silicon and glassy chalcogenides, Current Opinion in Solid State and Materials Science 51 (2001) 509-516.

[43] D. Drabold, Topics in the theory of amorphous materials, The European Physical Journal B 68 (2009) 1-21. 
[44] S. Roorda, C. Martin, M. Droui, M. Chicoine, A. Kazimirov, S. Kycia, Disentangling neighbors and extended range density oscillations in monatomic amorphous semiconductors, Phys. Rev. Lett. 108 (2012) 255501.

[45] R. Singh, S. Prakash, N. N. Shukla, R. Prasad, Sample dependence of the structural, vibrational, and electronic properties of a-Si:H: A densityfunctional-based tight-binding study, Physical Review B 70 (2004) 115213.

[46] A. R. Forouhi, I. Bloomer, Optical dispersion relations for amorphous semiconductors and amorphous dielectrics, Physical Review B 34 (1986) 7018-7026.

[47] S. Ruffell, K. Sears, A. P. Knights, J. E. Bradby, J. S. Williams, Experimental evidence for semiconducting behavior of Si-XII, Physical Review B 83 (2011) 075316.

[48] B. D. Malone, J. D. Sau, M. L. Cohen, Ab initio study of the optical properties of si-xii, Physical Review B 78 (2008) 161202(R).

[49] S. Wippermann, M. Vörös, D. Rocca, A. Gali, G. Zimanyi, G. Galli, Highpressure core structures of si nanoparticles for solar energy conversion, Physical Review Letters 110 (2013) 046804.

[50] J. S. Williams, J. S. Field, M. V. Swain, Mechanical property characterisation of crystalline, ion implantation amorphised and annealed relaxed silicon with spherical indenters, in: P. H. Townsend, T. P. Weihs, P. B. J. E. Sanchez, Jr. (Eds.), Thin Films: Stresses and Mechanical Properties IV, volume 308 of Materials Research Society Symposium Proceedings, Warrendale, PA, 1993, pp. 571-576.

[51] G. L. Olson, J. A. Roth, Kinetics of solid phase crystallization in amorphous silicon, Materials Science Reports 3 (1988) 1-77.

[52] D. Beeman, R. Tsu, M. F. Thorpe, Structural information from the Raman spectrum of amorphous silicon, Physical Review B 32 (1985) 874-878. 
[53] M. Marinov, N. Zotov, Model investigation of the Raman spectra of amorphous silicon, Physical Review B 55 (1997) 2938-2944.

[54] A. P. Sokolov, A. P. Shebanin, O. A. Golikova, M. M. Mezdrogina, Structural disorder and optical gap fluctuations in amorphous silicon, Journal of Physics: Condensed Matter 3 (1991) 9887-9894.

[55] J. Fortner, J. S. Lannin, Structural relaxation and order in ion-implanted Si and Ge, Physical Review B 37 (1988) 10154-10158.

[56] G. Morell, R. S. Katiyar, S. Z. Weisz, H. Jia, J. Shinar, I. Balberg, Raman study of the network disorder in sputtered and glow discharge a-Si:H films, Journal of Applied Physics 78 (1995) 5120-5125.

[57] R. F. Egerton, Electron Energy Loss Spectroscopy in the Electron Microscope, 2nd ed., Plenum, New York, 1996.

[58] D. R. McKenzie, D. Muller, B. A. Pailthorpe, Compressive-stress-induced formation of thin-film tetrahedral amorphous carbon, Physical Review Letters 67 (1991) 773.

[59] A. C. Ferrari, A. Libassi, B. K. Tanner, V. Stolojan, J. Yuan, L. M. Brown, R. S. E., B. Kleinsorge, J. Robertson, Density, $s p^{3}$ fraction, and cross-sectional structure of amorphous carbon films determined by X-ray reflectivity and electron energy-loss spectroscopy, Physical Review B 62 (2000-II) 11089-11103.

[60] S. Kivelson, C. D. Gellat, Jr., Effective-mass theory in noncrystalline solids, Physical Review B 10 (1979) 5160-5177.

[61] S. M. Sze, Physics of Semiconductor Devices, 2nd ed., Wiley-Interscience, 1981.

[62] A. Liu, R. Arenal, D. Miller, X. Chen, J. J. O. Erylmaz, A. Erdemir, J. Woodford, Structural order in near-frictionless dlc films probed at 
three different length scales in transmission electron microscope, Phys. Rev. B 75 (2007) 205402.

[63] L. Gorecki, A. C. Y. Liu, T. C. Petersen, High-resolution radial distribution function of pure ion-implanted amorphous silicon measured using tilted-illumination selected area electron diffraction., Microscopy and Microanalysis 20 (2014) 50-54.

[64] T. C. Petersen, W. McBride, D. McCulloch, I. K. Snook, I. Yarovsky, Refinements in the collection of energy filtered diffraction patterns from disordered materials, Ultramicrosc. 103 (2005) 175-283.

[65] D. R. G. Mitchell, T. C. Petersen, Rdftools: a software tool for quantifying short-range ordering in amorphous materials, Microsc. Res. Tech. 75 (2012) 153-163.

[66] K. Laaziri, S. Kycia, S. Roorda, M. Chicoine, J. L. Robertson, J. Wang, S. C. Moss, High-energy x-ray diffraction study of pure amorphous silicon, Phys. Rev. B 60 (1999) 13520.

[67] J. Fortner, J. S. Lannin, Radial distribution functions of amorphous silicon, Physical Review B 39 (1989) 5527-5530.

[68] J. F. Justo, M. Z. Bazant, E. Kaxiras, V. V. Bulatov, S. Yip, Interatomic potential for silicon defects and disordered phases, Phys. Rev. B 58 (1998) 2539 .

[69] J. Tersoff, Empirical interatomic potential for silicon with improved elastic properties, Phys. Rev. B 38 (1988) 9902.

[70] F. A. Stillinger, T. A. Weber, Computer simulation of local order in condensed phases of silicon, Phys. Rev. B 31 (1985) 5262.

[71] K. Nordlund, 2006. PARCAS computer code. The main principles of the molecular dynamics algorithms are presented in [99, 100]. The adaptive time step and electronic stopping algorithms are the same as in [101]. 
[72] H. Zhu, R. S. Averback, M. Nastasi, Molecular dynamics simulations of a 10 kev cascade in $\beta$-nial, Phil. Mag. A 71 (1995) 735.

[73] J. R. Dennis, E. B. Hale, J. Appl. Phys. 49 (1978) 1119.

[74] E. Holmström, A. V. Krasheninnikov, K. Nordlund, Quantum and classical molecular dynamics studies of the threshold displacement energy in si bulk and nanowire, in: D. Ila, J. K. N. Lindner, P. K. Chu, J. Baglin, N. Kishimoto (Eds.), Ion Beams and Nano-Engineering, volume 1181 of MRS Symposium Proceedings, MRS, Warrendale, PA, USA, 2009, pp. DD05-02.

[75] M. J. Norgett, M. T. Robinson, I. M. Torrens, A proposed method of calculating displacement dose rates, Nucl. Engr. and Design 33 (1975) $50-54$.

[76] H. J. C. Berendsen, J. P. M. Postma, W. F. van Gunsteren, A. DiNola, J. R. Haak, Molecular dynamics with coupling to external bath, J. Chem. Phys. 81 (1984) 3684.

[77] M. C. Payne, M. P. Teter, D. C. Allan, T. A. Arias, J. D. Joannopoulos, Iterative minimization techniques for $a b$ initio total-energy calculations: molecular dynamics and conjugate gradients, Rev. Mod. Phys. 64 (1992) 1045.

[78] X. Yuan, A. N. Cormack, Efficient algorithm for primitive ring statistics in topological networks, Comput. Mat. Sci. 24 (2002) 343.

[79] B. A. Weinstein, G. J. Piermarini, Raman scattering and phonon dispersion in Si and GaP at very high pressure, Physical Review B 12 (1975) $1172-1186$.

[80] T. Englert, G. Abstreiter, Determination of existing stress in silicon films on sapphire substrate using Raman spectroscopy, Solid-State Electronics 23 (1980) 31-33. 
[81] A. Kailer, Lokale Schädigung von Oberflächen und Phasenumwandlungen in harten, spröden Materialen verursacht druch mechanischen Kontakt, Shaker Verlag, Aachen, 1999.

[82] B. R. Lawn, A. G. Evans, A model for crack initiation in elastic/plastic indentation fields, Journal of Materials Science 12 (1977) 2195-2199.

[83] J. S. Custer, M. O. Thompson, D. C. Jacobson, J. M. Poate, S. Roorda, W. C. Sinke, Density of amorphous Si, Applied Physics Letters 64 (1994) $437-439$.

[84] B. Haberl, Structural Characterization of Amorphous Silicon, Ph.D. thesis, Australian National University, 2010.

[85] J. Nord, K. Nordlund, J. Keinonen, Amorphization mechanism and defect structures in ion-beam-amorphized Si, Ge, and GaAs, Physical Review B 65 (2002) 165329/1-14.

[86] J. C. Jamieson, Crystal structures at high pressures of metallic modifications of silicon and germanium, Science 139 (1963) 762-764.

[87] A. P. Gerk, D. Tabor, Indentation hardness and semiconductor-metal transition of germanium and silicon, Nature 271 (1978) 732-733.

[88] G. M. Pharr, W. C. Oliver, D. S. Harding, Electrical resistance of metallic contacts on silicon and germanium during indentation, Journal of Materials Research 6 (1991) 1129-1130.

[89] J. E. Bradby, J. S. Williams, M. V. Swain, In situ electrical characterization of phase transformations in Si during indentation, Physical Review B 67 (2003) 085205.

[90] R. H. Wentorf, Jr., J. S. Kasper, Two new forms of silicon, Science 139 (1963) 338-339. 
[91] R. O. Piltz, J. R. Maclean, S. J. Clark, G. J. Ackland, P. D. Hatton, J. Crain, Structure and properties of silicon XII: A complex tetrahedrally bonded phase, Physical Review B 52 (1995) 4072-4085.

[92] A. Kailer, K. G. Nickel, Y. G. Gogotsi, Raman microspectroscopy of nanocrystalline and amorphous phases in hardness indentations, Journal of Raman Spectroscopy 30 (1999) 939-946.

[93] J. E. Bradby, J. S. Williams, J. Wong-Leung, M. V. Swain, P. Munroe, Transmission electron microscopy observation of deformation microstructure under spherical indentation in silicon, Applied Physics Letters 77 (2000) 3749-3751.

[94] O. Graydon, Amorphous alternative, Nature Photonics 6 (2012) 716.

[95] R. A. Street, Hydrogenated Amorphous Silicon, Cambridge University Press, Cambridge, 1991.

[96] J. Robertson, Materials science: Crystallization of silicon ideas, Nature 418 (2002) 30.

[97] R. Rao, J. E. Bradby, J. S. Williams, Patterning of silicon by indentation and chemical etching, Applied Physics Letters 91 (2007) 123113/1-3.

[98] L. B. Aji, S. Ruffell, B. Haberl, J. Bradby, J. Williams, Correlation of indentation-induced phase transformations with the degree of relaxation of ion-implanted amorphous silicon, Journal of Materials Research 28 (2013) 1056.

[99] K. Nordlund, M. Ghaly, R. S. Averback, M. Caturla, T. Diaz de la Rubia, J. Tarus, Defect production in collision cascades in elemental semiconductors and fcc metals, Phys. Rev. B 57 (1998) 7556-7570.

[100] M. Ghaly, K. Nordlund, R. S. Averback, Molecular dynamics investigations of surface damage produced by kev self-bombardment of solids, Phil. Mag. A 79 (1999) 795. 
[101] K. Nordlund, Molecular dynamics simulation of ion ranges in the 1 - 100 kev energy range, Comput. Mater. Sci. 3 (1995) 448.

[102] W. Humphrey, A. Dalke, K. Schulten, Vmd - visual molecular dynamics, J. Molec. Graphics 14 (1996) 33-38.

\section{Appendix A. Experimental methods}

\section{Appendix A.1. Raman spectroscopy}

Significant differences in terms of peak position and width were observed between the different samples of a-Si after thermal annealing. The differences in peak positions can be quantified through detailed analysis of the Raman spectra. The main features used for the quantification are the half width at half maximum of the broad transverse optic-like $(\mathrm{TO}))^{6}\left(\Gamma_{T O} / 2\right)$, the intensity of the TO-like peak $\left(I_{T O}\right)$, and the intensity of the transverse acoustic-like (TA) peak $\left(I_{T A}\right)$. The characteristics of the TO-like peak are correlated with the vibrational bond-stretching modes and thus $\Gamma_{T O} / 2$ can indicate whether the state of a-Si is relaxed or unrelaxed [55, 21, 53. A simple empirical equation directly relates $\Gamma_{T O}$ to the RMS bond-angle $\Delta \theta$ as derived by Beeman et al. [52]:

$$
\Gamma_{T O}=15+6 \Delta \theta
$$

Note that usually $\Gamma_{T O} / 2$ is used for this analysis, since only the high-frequency side of the TO-like peak is fully optic, whereas the lower-frequency side of the TO-like peak overlaps with acoustic modes [53]. Consistent with previous work 21], $\Gamma_{T O} / 2$ was thus determined directly from the maximum of the TO-like peak and the minimum on the high-frequency side.

Information on the dihedral angle deviation and hence intermediate-range order (IRO) in the materials can be accessed by means of the intensity of the

\footnotetext{
${ }^{6}$ Note that the TO peak is a well defined, sharp peak in dc-Si. The peak in a-Si referred to as 'TO-like' represents its amorphous equivalent broadened due to breakdown of quantum confinement. Equally, the other broad a-Si peaks are only observed due to this breakdown. To signify this, the peaks are supplied with the suffix '-like' in the remainder of the text.
} 
TA-like peak. The vibrations giving rise to this peak are due to bond-bending modes, i.e., to the collective vibration of entire Si tetrahedra, and the peak intensity is related to the amplitude of fluctuations of the dihedral angle [54]. Hence, a more rigid, or more ordered, network will yield a lower TA-like peak intensity. In order to gain a normalized measure of the ordering of the network through this relationship, the ratio $I_{T A} / I_{T O}$ was calculated, with a lower ratio indicating a more ordered network [55, 56, 15]. For computing this ratio, the intensity of the TO-like peak used was the peak intensity determined for $\Gamma_{T O} / 2$ as described above. The intensity of the TA-like peak is determined from the maximum on the low-frequency side of the peak, as only this side is free from overlap with optic modes [53]. The intensity of the peak was obtained directly from the spectrum after fitting using the Savitzky-Golay formalism for 5-point smoothing using the Origin package.

Although less prominent in the relaxed PI case, in the as-indented PI sample the dc-Si transverse optic-like (TO) band is clearly split into two bands at $510 \mathrm{~cm}^{-1}$ and $528 \mathrm{~cm}^{-1}$. This behavior is presumably due to residual stresses in the underlying dc-Si introduced by the indentation. The band at $510 \mathrm{~cm}^{-1}$ is consistent with tensile stress, whereas the band at $528 \mathrm{~cm}^{-1}$ is suggestive of compressive stress [79, 80, 81]. The reason for the presence of two distinct bands rather than an integration over the entire dc-Si volume sampled might be the fact that residual stresses are directional. Therefore, it is likely that the tensile stress present in the underlying dc-Si is in the $x / y$-direction of the indenter geometry and thus a precursor to the formation of a median crack [82, whereas the compressive stress in the underlying dc-Si is in the $z$-direction of the indenter geometry.

Appendix A.2. Determining the mass density

The mass density $\rho_{0}$ was measured using electron energy loss spectroscopy (EELS) and the free-electron or "jellium" model. In this model, assuming each Si atom contributes four valence electrons, the plasmon energy $E_{p}$ and $\rho_{0}$ are 
related by [57]

$$
\rho_{0}=\frac{\epsilon_{0}}{4 \hbar^{2} N_{A} e^{2}} M_{S i} m^{*} E_{p}^{2}
$$

Here $\hbar$ is the reduced Planck constant, $N_{A}$ the Avogadro constant, $M_{S i}$ the atomic mass of $\mathrm{Si}, e$ the elementary charge, $\epsilon_{0}$ the permittivity of free space and $m^{*}$ the effective electron mass [57. Theoretical calculations demonstrate an equivalent $m^{*}$ for pure dc-Si and a-Si [60. Hence, the known longitudinal $m^{*}$ of 0.98 for dc-Si [61] was assumed for all Si forms. $E_{p}$ was directly measured by EELS in a Philips CM30T TEM working at $100 \mathrm{kV}$ by using a Gatan 666 PEEL spectrometer. The energy resolution of the EELS measurement was $\sim 1 \mathrm{eV}$. EELS spectra were recorded in diffraction mode using convergence and collection semiangles of 3 and $5 \mathrm{mrad}$, respectively. The determination of $E_{p}$ using the Richardson-Lucy algorithm for the deconvolution of the low-loss EELS was unsuccessful because of the low intensity of one data set. For consistency, $E_{p}$ was therefore determined from the low-loss spectrum after de-convolution of the zero-loss peak from the plasmon peak using the Fourier-Log method [57]. The plasmon peak was fitted using a DC offset as a dark-current correction and the energy-loss function [57] in an energy range of 13 to $22 \mathrm{eV}$. From the determined $E_{p}$, the mass density $\rho_{0}$ was then calculated using Eq. A.2.

Appendix A.3. Measuring and analyzing the radial distribution function

For measuring the radial distribution function (RDF) of the samples in the TEM, the ion-implanted specimens were polished using low-energy, glancingangle $\mathrm{Ar}^{+}$ions at low temperature for 10 minutes to remove any surface oxide (PIPS, $1 \mathrm{keV}, 2^{\circ},-100^{\circ} \mathrm{C}$ ) just prior to insertion into the TEM. The indented specimens were not ion-milled, as they were not mechanically (due to significant residual stresses) and thermally stable enough for the procedure. Selected-area diffraction patterns were obtained in a JEOL JEM-2100F FEGTEM using a Gatan UltraScan 1000 (2k x 2k) CCD camera. The dark-field tilt was employed to tilt the incident beam and bring diffracted intensity associated with different ranges of the scattering vector onto the CCD camera, thereby extending the total accessible angular range. By splicing the diffraction patterns 
together, diffracted intensities were measured out to a maximum scattering vector $s=2 \sin (\theta) / \lambda$ of 3.3-3.7 $\AA^{-1}$ for the ion-implanted samples, permitting a high-resolution RDF to be measured as detailed in Refs [63, 64, 65]. For the $\mathrm{PI}$ a-Si, the maximum angular range was $2.8-3.0 \AA^{-1}$, since the sufficiently thin indented regions provided a limited field of view (i.e., due to the fact the PI a-Si can only be created in localized volumes of a maximum of $10 \mu \mathrm{m}$ diameter). The diffracted intensity was radially averaged into $I(s)$ and transformed to the reduced intensity function $\phi(s)$ using tabulated values for the atomic scattering factor 63. The reduced intensity function was then Fourier-transformed to a reduced RDF, $G(r)=J(r) / r-4 \pi r \rho_{0}$, where $\rho_{0}$ was determined using EELS as detailed above and $J(r)$ is the RDF [41]. Parameters associated with the SRO of the materials were extracted from the RDF.

Coordination numbers were measured by integrating each RDF between the limits of $2.1 \AA$ and $3.0 \AA$. For the ion-implanted a-Si, the error in the values quoted is the min-max spread as the high integration limit was varied between 2.9 and $3.1 \AA$. For the PI a-Si, the min-max spread was estimated from different regions probed from each specimen, as opposed to the variation of the integration limits. For electron diffraction, truncation with a finite $Q_{\max }$ results in a broadening that can be approximated by a Gaussian and does not affect the coordination number if suitable integration limits were chosen. The maxima of the first and second-nearest-neighbor peaks were used to determine the average bond-angle $\langle\theta\rangle \underline{63}$ as a statistical measure of both structure and implicit correlations due to bonding energetics. The bond-angle spread was measured by fitting the left-hand shoulder of the second-nearest-neighbor peak with a Gaussian and deconvoluting the experimental resolution function to obtain the natural width of the peak. For the PI a-Si, three areas were sampled for each specimen. Bond-angle and coordination statistics were averaged for each specimen and the variability was then estimated from the min-max spread.

The bond-angle distortion $\Delta \theta$ appears to depend on the spread of the secondnearest-neighbor peak due to thermal and instrumental broadening. While the effects of thermal broadening alone are small, an upper limit for instrumental 
broadening and consequent deconvolution had to be estimated. The rigidity of the strong covalent $\mathrm{Si}-\mathrm{Si}$ bond implies a very sharp first diffraction peak approximating a Dirac delta function. The broadening of this first peak can be regarded as an extreme upper limit for the 'response function' and cannot be smaller than the true broadening. Since amorphous covalent networks exhibit a real dispersion of nearest-neighbor distances, which contributes to the broadening, approximating the true broadening by this width is most likely a severe overestimation. Nonetheless, Gaussian fits to the first diffraction peak of all RDFs were made to provide such an overestimate of the inherent broadening of all second diffraction peaks. This was then subtracted in quadrature for deconvolution [67. This lowered the values for the average bond-angle distortion $\Delta \theta$ by $\sim 4-9 \%$. The resulting values further departed from $\Delta \theta$ measured by Raman in terms of absolute values and also trends. Consequently, further broadening effects do not seem to affect measurement of $\Delta \theta$ from our RDFs, and deconvolution of the experimental resolution function was deemed sufficient.

\section{Appendix B. Simulation methods}

Appendix B.1. Creating the structural ensembles of a-Si

Ion implantation

To produce a structural ensemble of $\mathrm{Si}$ amorphized through ion implantation, we performed MD simulations [71] of the amorphization of the material using $100 \mathrm{eV}$ self-recoils within a periodic simulation cell of approximately the dimensions $9 \mathrm{~nm} \times 9 \mathrm{~nm} \times 9 \mathrm{~nm}$. The pristine crystal structure at a temperature of $77 \mathrm{~K}$ was used as the initial structure for these simulations. First, a single recoil event was simulated, letting the simulation run for a total of $3 \mathrm{ps}$, during which the simulation cell was cooled back down to $77 \mathrm{~K}$. The final structure from this simulation, translated randomly over the periodic boundaries, was then used as the initial structure for the next recoil, and successive recoils were thus simulated until the total kinetic energy deposited in the cell was $\sim 35 \mathrm{eV} /$ atom for runs with the EDIP potential and $\sim 55 \mathrm{eV} /$ atom with the Tersoff potential. These doses were selected based on the angular structure factor analysis [72]. 
The structure was considered to be amorphous when this factor did not change anymore with increasing dose. Comparison with an average experimental value of amorphization dose obtained from several different ion irradiation studies of about $12 \mathrm{eV} /$ atom [73] shows that these simulated doses are consistent with dose levels where also experimentally $\mathrm{Si}$ is fully amorphized. A lower number of recoils was sufficient to accomplish amorphization for EDIP than for Tersoff, partly because of the lower threshold displacement energy of the former [74]. Using the interatomic-potential specific displacement energies $E_{d}$ of $16 \mathrm{eV}$ and $19 \mathrm{eV}$ and the NRT-dpa equation [75], $N_{F P}=0.8 E_{\text {damage }} / 2 E_{d}$, where $N_{F P}$ is the number of Frenkel pairs produced by a recoil of kinetic energy $E_{\text {damage }}$, one finds displacements-per-atom (dpa) values of $N_{F P}=2.5$ and 2.1 for EDIP and Tersoff, respectively. We also used cells with a total energy deposition of $\sim 10 \%$ of the above values to produce a separate line of structural ensembles in the manner detailed below. At these lower doses, some small amount of crystalline structure was still detectable in the cells. A total of 30 cells of a-Si were created using this method with each interatomic potential and ion dose. Next, the cells were annealed for a duration of $1 \mathrm{~ns}$ at $723 \mathrm{~K}$ and subsequently slowly cooled back down to $77 \mathrm{~K}$. We also used cells prior to annealing to produce a separate set of ensembles, in the way detailed below. The Berendsen thermostat and barostat [76] were used to control the temperature and to relax the dimensions of the orthogonal simulation box in order to attain zero total pressure in all MD runs throughout the work.

An ensemble of structures was then generated for each potential, dose, and heat treatment. For each structure, two simulation cells were chosen at random among the 30 generated. The two cells were rotated randomly in steps of $90^{\circ}$ in all dimensions, translated randomly over the periodic boundaries, and then joined along one face of each cell to produce a stacked structure of dimensions $9 \mathrm{~nm} \times 9 \mathrm{~nm} \times 18 \mathrm{~nm}$. Conjugate gradient relaxation [77] was then used to relax the newly formed stacked structure, as unphysically close atom pairs are prone to form in such a joining procedure. Finally, using another MD simulation, the cell was brought from a temperature of $0 \mathrm{~K}$ to $77 \mathrm{~K}$ at a rate of $0.01 \mathrm{~K} / \mathrm{fs}$ and 
then held at $77 \mathrm{~K}$ for a duration of $50 \mathrm{ps.} \mathrm{The} \mathrm{entire} \mathrm{procedure} \mathrm{was} \mathrm{repeated}$ until an ensemble of 200 structures of implanted a-Si had been produced using each interatomic potential, implantation dose, and either with or without the 1 ns annealing procedure following the implantation. The only ensemble to match the experimental SRO and density data to a satisfactory degree was the one constructed from the full dose of implantation with the $1 \mathrm{~ns}$ annealing run, using the EDIP potential.

Quenching from the melt

Next, we simulated amorphization of Si by melting and quenching. Starting from a configuration of silicon atoms that was constructed by selecting points randomly in space using only the constraint that a distance smaller than $1.8 \AA$ between any two atoms was prohibited, we first equilibrated a liquid cell of $9 \mathrm{~nm} \times 9 \mathrm{~nm} \times 9 \mathrm{~nm}$ at $T=2000 \mathrm{~K}$ by running a simulation of $50 \mathrm{ps}$ of duration at that temperature. Then, the system was quenched down to $77 \mathrm{~K}$ at a rate $0.01 \mathrm{~K} / \mathrm{fs}$, and finally kept at that temperature for $60 \mathrm{ps}$. A total of 200 structures were created with this simulation setup for each interatomic potential. As with the implanted ensembles, we then proceeded to join and relax 200 randomly chosen pairs of simulation cells to create an ensemble of 200 structures of the dimensions $9 \mathrm{~nm} \times 9 \mathrm{~nm} \times 18 \mathrm{~nm}$ using each interatomic potential.

\section{Bond-switching Monte Carlo}

We then proceeded to create an ensemble of structures by starting from a set of perfect CRN models and then further relaxing the networks through MD. To create the CRN models, we employed the bond-switching Monte Carlo algorithm of Wooten, Winer, and Weaire 29, as implemented for the simplified Si-Si Keating potential by von Alftan et al. 31 In this procedure, we first created a total of 200 annealed CRN structures of the dimensions $3 \mathrm{~nm} \times 3 \mathrm{~nm}$ $\times 19 \mathrm{~nm}$, using the same annealing parameters as in Ref. 31] Then, using the EDIP and the Tersoff potential in turn, each CRN structure was further relaxed by bringing the cell from a temperature of $0 \mathrm{~K}$ to $77 \mathrm{~K}$ at a rate of $0.01 \mathrm{~K} / \mathrm{fs}$ 
and then keeping the system at $77 \mathrm{~K}$ for a duration of $50 \mathrm{ps}$. Using this method, 200 structures were created for each interatomic potential.

\section{Paracrystalline model}

Finally, we created the following two paracrystalline ensembles. Through cutting out uniformly distributed spherical regions in the WWWMD EDIP and WWWMD Tersoff structures and subsequently inserting into each of these voids a randomly rotated perfectly ordered dc-Si crystallite of diameter $\sim 1 \mathrm{~nm}$, a set of 200 paracrystalline structures with $\sim 10 \%$ of the total volume being crystalline was created for each potential. We then relaxed each structure using conjugate gradients and MD, analogously to the approach for our implanted and meltquenched simulation cells. The resulting grains inside the amorphous structure are indeed highly distorted, yet bearing a clear resemblance to dc-Si.

An example of a structure in the Implanted EDIP ensemble and the WWWMD Tersoff ensemble is visualized in Fig. B.7.

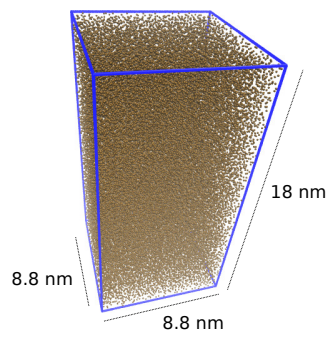

(a)

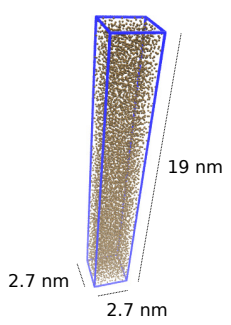

(b)

Figure B.7: Visualization 102 of a structure in the Implanted EDIP (a) and the WWWMD Tersoff (b) ensemble.

Appendix B.2. Analyzing the atomistic models

For quantifying the SRO characteristics of the created atomistic models of a-Si, we first computed the pair-distribution function $g(r)=\rho(r) /(N / V)$ [4] for each structure in each ensemble, where $\rho(r)$ is the average number density of atoms at radius $r$ from any given atom, and $N / V$ is the total number density of the structure. We then averaged $g(r)$ over all structures in each ensemble. 
After this, for direct comparison with experiment, we computed the reduced RDF $G(r)$, similarly averaged over all structures in each ensemble. We then determined the mean nearest-neighbor coordination number $Z$ of each ensemble by performing the integral

$$
\langle Z\rangle=\frac{N}{V} \int_{0}^{r_{\min }} 4 \pi r^{2} g(r) d r
$$

where $r_{\min }$ is the radius corresponding to the first minimum in $g(r)$ after the first peak. By examining $g(r)$ for each ensemble, a universal cutoff of $r=2.8 \AA$ was found to just encompass the nearest-neighbor coordination shell. Hence, any pair of atoms with a distance equal to or smaller than this were considered bonded in all subsequent analysis. The distribution of coordination numbers was then determined for each ensemble, by simply employing this cutoff value. Additionally, we calculated the distribution of bond-angles $\theta$ in each ensemble, and from that the mean bond-angle $\langle\theta\rangle$ and the RMS bond-angle deviation $\Delta \theta$. We also computed the mass density of each ensemble with respect to that of dc-Si $\left(\rho_{0} / \rho_{\text {crystal }}\right)$. Next, in order to assess the overall stability of the structures, we calculated the difference in potential energy per atom relative to dc-Si $\left(E-E_{c}\right)$. Finally, in order to access a quantitative measure of IRO present in the structures, we determined distributions of primitive rings in each ensemble using the method of Yuan et al. 78, as well as the distribution of dihedral angles [40. The latter were analyzed further by fitting the peaks with two Gaussians using the Origin package to obtain peak maxima. 